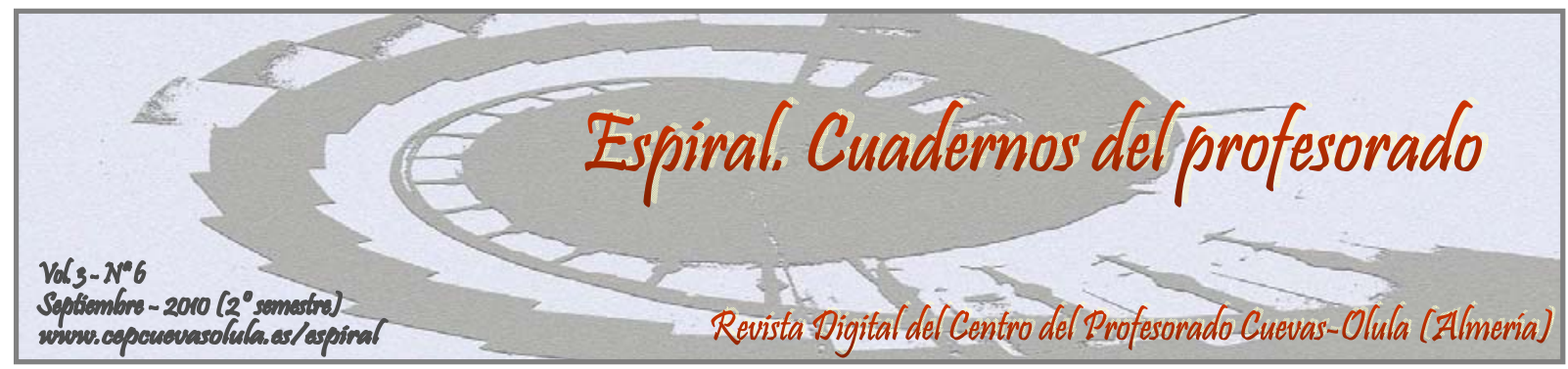

\title{
INNOVACIÓN DOCENTE E IDENTIFICACIÓN DE INHIBIDORES DEL APRENDIZAJE EN EL ÁREA DE EMPRESA. UNA PROPUESTA METODOLÓGICA
}

\author{
TEACHING INNOVATION AND IDENTIFICATION OF LEARNING INHIBITORS \\ IN THE BUSINESS AREA. A METHODOLOGICAL PROPOSAL
}

\section{Verónica Baena Graciá}

\section{Departamento de Empresa de la Universidad Europea de Madrid, España}

RESUMEN: El siglo XXI está marcado por un entorno cambiante que nos obliga a adaptarnos continuamente. La competencia y competitividad empresarial es elevada y por ello las universidades deben asegurarse de que sus alumnos no sólo adquieren los conocimientos teóricos necesarios sino además, desarrollan determinadas competencias que garantizan el éxito a largo plazo. Este trabajo identifica algunos de los factores que inhiben el aprendizaje en los alumnos universitarios y describe una práctica integradora de conocimientos denominada "La Fábrica de Cartas" que minimiza el efecto de dichos factores. Los resultados obtenidos nos permite concluir que "La Fábrica de Cartas" contribuyó a que los alumnos de cuarto curso de la Licenciatura en Administración y Dirección de Empresas (ADE) y doble licenciatura con ADE asimilaran mejor los conocimientos teóricos adquiridos en diferentes asignaturas vinculadas al área de empresa (marketing, producción y dirección de empresas). Asimismo, la actividad coadyuvó al desarrollo por parte de los alumnos de diferentes habilidades comunicativas y competencias básicas para el mundo profesional, tales como el trabajo en equipo, gestión de la información, planificación, innovación, creatividad y responsabilidad, entre otras.

Palabras clave: Innovación docente, inhibidores del aprendizaje, Espacio Europeo de Educación Superior, aprendizaje activo.

ABSTRACT: The XXI century is characterized by an evolving environment that requires continuous adaptation. Both business competition and competitiveness are high and therefore, universities have to be sure their pupils not only acquire theoretical knowledge but also develop certain skills that guarantee long term success. This work identifies some factors that may constrain or inhibit the learning process in university students and describes a practice called "The Card Manufacturing Game", which minimizes the effect of those inhibitors. Results help us to conclude that "The Card Manufacturing Game" may assist senior students of Business Bachelor Degree to improve the way they assimilate the theoretical concepts acquired in different business courses (marketing, operation and business management). Moreover, this learning practice helped the students to develop several valued skills in the professional environment, such as team working, knowledge management, planning, innovation, and responsibility, among others.

Key words: Teaching innovation, learning inhibitors, integrative knowledge practice, active learning. 
Baena Graciá, V. (2010). Innovación docente e identificación de inhibidores del aprendizaje en el área de empresa. Una propuesta metodológica. Espiral. Cuadernos del Profesorado [en línea], 3(6), 3-14. Disponible en: http://www.cepcuevasolula.es/espiral.

Fecha de recepción: 13/05/2010

Fecha de aceptación: 07/07/2010
Enviar correspondencia a: veronica.baena@uem.es

\section{1.- INTRODUCCIÓN.}

Tomando como punto de partida la definición que Pagani y González (2002) realizan de los créditos ECTS - European Credit Transfer and Accumulation System - (Sistema Europeo de Transferencia y Acumulación de Créditos, en español), podemos decir que se trata de una unidad de valoración de la actividad académica medida a partir del volumen de trabajo real que el estudiante realiza para superar cada una de las asignaturas. Es decir, se trata de un concepto en el que se integra tanto la enseñanza teórica y práctica como el resto de actividades académicas dirigidas por el profesor.

En este sentido, tal y como señalan Cuestas, Fenollar y Román (2008) esta nueva forma de medir el esfuerzo del alumno implica la modificación del concepto tradicional de enseñanza, de manera que se consideren dos aspectos no tenidos en cuenta por la anterior regulación de los créditos. En primer lugar, el volumen de trabajo real (esfuerzo del alumno) y en segundo lugar, la superación de las asignaturas (resultado académico).

Además, los cambios a los que nos enfrentamos en la educación superior en Europa y sus efectos sobre las condiciones de un mercado laboral cada vez más competitivo, hacen necesario que las universidades se esfuercen en conocer a su alumnado. Sólo así será posible que las universidades sean capaces de adaptar sus programas formativos Gibbs (2007) y las metodologías docentes empleadas en sus nuevos títulos de grado y posgrado (Barber, 2008; Pusztai y Szabó, 2008).

En consecuencia, la implantación del nuevo sistema universitario exige cambiar la actual dinámica de enseñanza por otra donde los estudiantes asuman responsabilidades, dejen de ser sujetos pasivos para convertirse en los protagonistas de su propio aprendizaje (Whitehead, 2008) y por tanto, estén preparados para los cambios que se producen en su entorno social y profesional (De Juan et al., 2008).
Por otra parte, no es poca la literatura que señala que los estudiantes universitarios aparte de conocer en profundidad los contenidos de las asignaturas que cursan, deben desarrollar determinadas competencias que les ayuden a desenvolverse en un entorno de trabajo cambiante, competitivo y complejo (Hunt, Eagle y Kitchen, 2004, entre otros).

En este sentido, los programas de educación universitaria tienen que garantizar el desarrollo de determinadas habilidades sociales puesto que lejos de implicar un debilitamiento de la enseñanza tradicional, la inclusión de las habilidades sociales en los currículos educativos es un complemento indispensable para el desarrollo cognitivo (Delors, 1998).

Por ello, tal y como señala López (2008), sólo los planes de estudio que potencian un equilibrio entre los conocimientos teóricos y las habilidades sociales son capaces de formar estudiantes capaces de afrontar y gestionar soluciones integrales mediante la aplicación de habilidades de negociación, trabajo en equipo, asertividad y manejo de conflictos; características ampliamente valoradas en el mundo profesional al que posteriormente se integrarán.

En otras palabras, los profesores universitarios no deben limitarse a la mera transmisión de conocimiento a su alumnado sino que por el contrario, han de contribuir al desarrollo competencial de sus alumnos y asegurarse de que éstos son capaces de aplicar dichas competencias en el mundo laboral (Canzer, 1997). Asimismo, algunos trabajos demuestran lo difícil que puede resultar adquirir determinadas competencias, tales como el liderazgo, gestión de personas, distribución de tareas (Adrian y Palmer, 1999) o el trabajo en equipo (Wright, Bitner y Zeithaml, 1994) si el alumno se limita a seguir un aprendizaje meramente pasivo.

Sin embargo, es relativamente escaso el número de trabajos que han analizado este aspecto y la mayoría de los que lo han hecho se han centrado en el sistema educativo anglosajón 
(Elliot y Shin, 2002; Hunt, Eagle y Tchen, 2004; Meterissian, Liberman y McLeod, 2007; Christensen, Horn y Johnson, 2008), lo que dificulta su extrapolación al entorno del Espacio Europeo de Educación Superior (EEES).

El objetivo del presente trabajo es cubrir este vacío. Para ello, se describe una actividad docente llevada a cabo en una universidad española que ayudó a los alumnos de tercer y cuarto curso de la Licenciatura en Administración y Dirección de Empresas (ADE) en el desarrollo de varias competencias, a la vez que les sirvió para asimilar diferentes conocimientos teóricos adquiridos a lo largo de la carrera.

En concreto, la práctica llevada a cabo integró diferentes conceptos relacionados con las áreas de marketing, producción y gestión empresarial ya que numerosos estudios remarcan la importancia de coordinar dichas funciones para conseguir el éxito empresarial (Barreiro et al, 2004, Baena et al., 2008, entre otros).

De este modo, se pretende contribuir a la literatura a través del diseño y puesta en práctica de una actividad docente que facilite a los alumnos la integración de algunos aspectos básicos de la empresa, a la vez que les ayude en el desarrollo de diferentes competencias necesarias para desenvolverse con soltura en el mundo profesional, tales como el trabajo en equipo, integración de la información, planificación, innovación, habilidades comunicativas, creatividad y responsabilidad, entre otras.

Para lograr los objetivos antes expuestos, en el siguiente apartado se describe la actividad denominada "La Fábrica de Cartas". Asimismo, se expone la metodología seguida en nuestra investigación y la muestra de alumnos con la que se ha trabajado, para seguidamente explicar los resultados obtenidos. Por último, este artículo finaliza con un apartado destinado a resumir las principales ideas del trabajo, así como las limitaciones y futuras líneas de investigación en el área de innovación educativa.

\section{2.- METODOLOGÍA.}

\section{La Actividad: La Fábrica de Cartas.}

Tal y como se ha expuesto en la sección anterior, este trabajo pretende contribuir a la mejora y adaptación docente exigida desde los planes de estudios adaptados al EEES. El primer paso consistió en la identificación de los factores que inhiben el aprendizaje entre los alumnos universitarios. Sólo así, seremos capaces de proponer una tarea docente que minimice estos efectos y mejore la asimilación de los conceptos teóricos adquiridos por el alumnado en clase, a la vez que les permita avanzar en su desarrollo competencial.

En este sentido, los autores recurrieron a su experiencia docente así como a la literatura previa (véase, por ejemplo, Elliott y Shin, 2002; Norman y Hyland, 2003; Bullock y Muschamp, 2006; Shu-Hui y Smith, 2008, García-Aracil, 2009) para la identificación de los inhibidores del aprendizaje. Como resultado nueve variables fueron seleccionadas.

A continuación, se repartió un cuestionario semi-estructurado (disponible en el Anexo de este trabajo) a todos los alumnos de último curso de la Licenciatura de Administración y Dirección de Empresas (ADE) y de la doble licenciatura con ADE (ADE + Investigación y Técnicas de Mercado, ADE + Publicidad, ADE + Ingeniería Industrial, $\mathrm{ADE}$ + Ingeniería Informática y ADE + Ingeniería de Telecomunicaciones) impartida en la universidad española donde trabajan los autores. En dicho cuestionario se les solicitaba que evaluasen del 1 (muy en desacuerdo) al 5 (muy de acuerdo) su opinión acerca del modo en que cada una de las siguientes variables dificulta el proceso de aprendizaje:

1. La asignatura es aburrida.

2. La asignatura es difícil.

3. El profesor es aburrido.

4. El material utilizado en clase es aburrido.

5. El estudiante está cansado.

6. El estudiante no percibe la utilidad práctica de la asignatura.

7. El estudiante no tiene bastante tiempo para estudiar fuera de clase.

8. El profesor no es claro en sus explicaciones.

9. Al estudiante le resulta complicado concentrarse en clase.

En total, se recogieron un total de 692 formularios debidamente cumplimentados y se garantizó el anonimato. En este sentido, es preciso señalar que el tamaño muestral obtenido es suficientemente representativo ya que superó el número de observaciones necesarias para que la muestra resultase precisa a un nivel de confianza del $99 \%$ con respecto al tamaño de la 
Tabla 1: Estadísticos Descriptivos - Primer Cuestionario

\begin{tabular}{cccccccccc}
\hline & VAR01 & VAR02 & VAR03 & VAR04 & VAR05 & VAR06 & VAR07 & VAR08 & VAR09 \\
\hline Media & 2,7917 & 2,4694 & 2,7708 & 2,7347 & 2,8542 & 2,8980 & 2,9583 & 2,8980 & 2,7959 \\
\hline Dv. típ. & 1,0097 & 0,7389 & 1,3407 & 0,9953 & 1,1297 & 1,0848 & 1,2876 & 1,0848 & 1,0404 \\
\hline Mínimo & 1,0000 & 1,0000 & 1,0000 & 1,0000 & 1,0000 & 1,0000 & 1,0000 & 1,0000 & 1,0000 \\
\hline Máximo & 5,0000 & 5,0000 & 5,0000 & 5,0000 & 5,0000 & 5,0000 & 5,0000 & 5,0000 & 5,0000 \\
\hline
\end{tabular}

población: un total 911 alumnos de $4^{\circ}$ curso de $\mathrm{ADE}$ y de la doble licenciatura con $\mathrm{ADE}^{1}$.

A través del análisis de los estadísticos descriptivos recogidos en la Tabla 1 sobre los nueve inhibidores del aprendizaje incluidos en el cuestionario, podemos observar que más de la mitad de los alumnos encuestados consideraron que la totalidad de los factores anteriormente expuestos dificultaban su aprendizaje. El porcentaje oscila entre el 49 por ciento y el 59 por ciento, dependiendo del factor sobre el que se preguntaba: i) la asignatura es aburrida (56\%); ii) la asignatura es difícil (49\%); iii) el profesor es aburrido (55\%); iv) el material utilizado en clase es aburrido (55\%); v) el estudiante está cansado $(57 \%)$; vi) el estudiante no percibe la utilidad práctica de la asignatura (58\%); vii) el estudiante no tiene bastante tiempo para estudiar fuera de clase (59\%); viii) el profesor no es claro en sus explicaciones (58\%); y finalmente, ix) al estudiante le resulta complicado concentrarse en clase $(56 \%)$.

Para estar seguros de la consistencia interna del cuestionario, se llevó a cabo un análisis de correspondencia múltiple. Tal y como se recoge en la Tabla 2, los resultados obtenidos mostraron la existencia de dos dimensiones. La primera de ellas está formada por las variables 1, 2, 3, 4, 5 y 9 mientras que las tres variables restantes $(6,7 \mathrm{y}$

${ }^{1}$ El lector interesado en obtener mayor información al respecto puede consultar Hair y otros (1999) y aplicar la siguiente fórmula:

$n=\frac{N * Z^{2}{ }_{\alpha} p * q}{d^{2} *(N-1)+Z_{\alpha}^{2} * p * q}$

donde:

$\mathrm{N}=$ Total de la población

$\mathrm{Z} \alpha 2=1.962$ (si la seguridad es del 99\%)

$\mathrm{p}=$ proporción esperada

$\mathrm{q}=1-\mathrm{p}($ en este caso $1-0.01=0.99)$

$\mathrm{d}=$ precisión (en este caso deseamos un $1 \%$ ).
8) componen la segunda dimensión. El Alfa de Cronbach asociado a cada una de las dimensiones es 0,857 y 0,781 respectivamente, lo que permitió descartar la existencia de problemas de multidimensionalidad y por lo tanto, falta de confiabilidad $^{2}$.

Asimismo, es preciso indicar que junto a las nueve preguntas cerradas antes expuestas, el cuestionario incluía dos preguntas abiertas. La primera de ellas solicitaba a los alumnos que indicasen el tipo de actividades que prefieren en el aula. En este sentido, los encuestados mostraron una abrumadora preferencia por las clases prácticas realizadas en grupo dentro del aula $(78 \%)$. Un porcentaje menor $(16 \%)$ apuntó su preferencia por los videos y otras actividades académicas de contenido lúdico. Junto a ello, el $6 \%$ restante señalaron que no les gustaba ningún tipo de actividad docente. Respecto a la segunda pregunta abierta incluida en el cuestionario - en la cual se solicitaba a los alumnos que señalasen el tipo de actividad que consideraban más efectiva en su proceso de aprendizaje -, la inmensa mayoría señaló que debía tratarse de una tarea con elevado contenido práctico y que les permitiese interactuar tanto con el profesor como con el resto de sus compañeros.

En resumen, los resultados obtenidos permitieron constatar la necesidad de poner en práctica una actividad que eliminase o al menos minimizase los factores previamente identificados como inhibidores del aprendizaje. Así pues, debía tratarse de una actividad que fuese capaz de garantizar el aprendizaje del alumno incluso en el caso en que éste considerase que la asignatura era aburrida y/o difícil, que el profesor no fuera claro en sus explicaciones y/o

${ }^{2}$ El coeficiente $\alpha$ de Cronbach (Cronbach, 1951) es uno de los más utilizados para medir la confiabilidad de una escala. Un valor del coeficiente inferior a 0,7 indica, por lo general, una baja consistencia interna (Peterson, 1994; Hair et al., 1999, entre otros), es decir, que la escala no mide un solo fenómeno sino varios y, por lo tanto, puede no ser apropiada para los fines de la investigación. 
fuese aburrido, que el material utilizado en clase fuese aburrido, que el alumno estuviese cansado, no percibiese la utilidad práctica de la asignatura, y/o no tuviese tiempo para estudiar fuera de clase y finalmente, que al estudiante le resultase difícil concentrarse en clase. Además, para garantizar el éxito de dicha actividad, era preciso que impulsase a los alumnos a desarrollar un papel activo en su proceso de aprendizaje, a la vez que les permitiese trabajar en equipo dentro del aula e interactuar con el resto de compañeros y el profesor responsable de la asignatura. El resultado fue la creación de "La Fábrica de Cartas", actividad docente que se describe a continuación.

\section{Reglas de la Actividad}

En "La Fábrica de Cartas", los alumnos son los responsables del funcionamiento de una fábrica que comercializa cartas de la baraja francesa cuyos moldes y materia prima son entregados por el profesor responsable de tutelar dicha actividad. La práctica se realiza durante una sesión de dos horas de clase que comprende 20 períodos de 2 minutos cada uno durante los cuales, los alumnos forman grupos (cada grupo es una fábrica de cartas) en los que deben tomar decisiones relativas al número de unidades óptimo que se deben fabricar, cómo hacerlo, a qué precio venderlas y a quién - mercado ordinario versus mercado secundario -. Además, cada grupo de alumnos debe tomar decisiones relativas al número de empleados de su empresa, especialización/estandarización en sus procesos productivos, fidelización de clientes, personalización de la oferta, diseño, fijación de precios, lanzamiento de promociones, etc.

Las reglas de la actividad son las siguientes:

- Cada empresa dispone de un presupuesto inicial de $100 €$;

- La materia prima (una hoja de papel) cuesta $10 €$ (los moldes, las tijeras y los rotuladores para dibujar las cartas son gratis);

- La mano de obra cuesta $1 €$ por empleado cada período;

- Las cartas vendidas son pagadas según su calidad: los alumnos saben que el mercado está dispuesto a pagar $30 €$ como máximo por cada carta de buena calidad (aquellas que han sido cortadas con tijeras y no a mano, presentan buena calidad del dibujo, etc.). Por el contrario, el mercado no pagará más de $15 €$ por cada carta de calidad regular (mal recortada, contorno defectuoso, etc.). Las cartas de baja calidad no son compradas. Las tentativas de engaño (por ejemplo, tratar de vender una carta de calidad regular como si fuera de calidad alta) tienen una penalización de $10 €$ para cada empresa;

- Iniciada la actividad, el mercado tardará 5 minutos antes de empezar a hacer sus pedidos, permitiendo una eventual creación de un stock mínimo por parte de cada fábrica. Pasado esos 5 minutos se realizará un pedido cada 120 segundos (hasta completar un total de 20 pedidos). No obstante, los alumnos no conocen el número de pedidos de los que se compone la actividad para evitar que dejen de producir en determinado momento para consumir el stock generado;

- La demanda del mercado ordinario varía de 0 a 8 cartas por pedido. A su vez, la demanda puede tratarse de ases de corazones, picas, rombos o tréboles. El profesor responsable de tutelar la actividad es el gestor de la demanda de mercado y actúa como único cliente, asistido en su caso, por uno o dos alumnos que le ayuden en su tarea de selección y compra de las mejores cartas al mejor precio. Una vez adquiridas las cartas demandadas en cada período (por ejemplo, en el período 6 se demandan dos ases de rombos), el mercado no realizará ningún pedido hasta pasados los 120 segundos de dicho período, lo que contribuye a una eventual formación de stock por parte de las empresas fabricantes de cartas;

- El layout ${ }^{3}$ de la fábrica será definido por cada grupo de alumnos;

- La actividad se interrumpe cada 6 períodos y cada una de las fábricas de cartas (grupo de alumnos) que participa en la actividad tiene la oportunidad de conocer su situación financiera (cuánto dinero tiene de beneficios o pérdidas) y la de sus competidores. Las empresas con pérdidas no pueden seguir operando en el mercado salvo que vendan su stock o materias primas en un mercado secundario (a otros

${ }^{3}$ El layout de una empresa se define como la ubicación o disposición de las diferentes categorías (producción, maquetado, transporte, etc.) en el proceso productivo y/o de venta de una empresa. 
Tabla 2: Resultados del Análisis de Correspondencias asociado al Primer Cuestionario.

\begin{tabular}{|c|c|c|c|}
\hline \multicolumn{4}{|c|}{ Historial de iteraciones } \\
\hline \multirow{2}{*}{$\begin{array}{c}\text { Número de } \\
\text { iteraciones }\end{array}$} & Varianza explicada & \\
\cline { 2 - 3 } & Total & Incremento & Pérdida \\
\hline $22^{\mathrm{a}}$ & 3,740 & 0,000 & 5,263 \\
\hline
\end{tabular}

a. Se ha detenido el proceso de iteración debido a que se ha alcanzado el valor de la prueba para la convergencia.

\begin{tabular}{|c|c|c|c|}
\hline \multirow{2}{*}{} & \multirow{3}{*}{ Resumen del modelo } \\
\cline { 3 - 4 } Dimensión & Alfa de & \multicolumn{2}{|c|}{ Varianza explicada } \\
\cline { 3 - 4 } & Cronbach & $\begin{array}{c}\text { Total } \\
\text { (Autovalores) }\end{array}$ & Inercia \\
\hline 1 & $\mathbf{0 , 8 5 7}$ & 4,205 & 0,467 \\
2 & $\mathbf{0 , 7 8 1}$ & 3,269 & 0,363 \\
Total & & 7,473 & 0,83 \\
Media &, $824^{\mathrm{a}}$ & 3,737 & 0,415 \\
\hline
\end{tabular}

a. El Alfa de Cronbach Promedio está basado en los autovalores promedio.

\begin{tabular}{|c|c|c|c|}
\multicolumn{4}{|c|}{ Medidas de discriminación } \\
\cline { 2 - 3 } & \multicolumn{2}{|c|}{ Dimensión } & \multirow{2}{*}{ Media } \\
\cline { 2 - 3 } & 1 & 2 & 0,461 \\
VAR01 & $\mathbf{0 , 6 1 9}$ & 0,302 & 0,344 \\
VAR02 & $\mathbf{0 , 4 0 4}$ & 0,284 & 0,601 \\
VAR03 & $\mathbf{0 , 6 4 1}$ & 0,562 & 0,45 \\
VAR04 & $\mathbf{0 , 4 9 5}$ & 0,405 & 0,182 \\
VAR05 & $\mathbf{0 , 2 7 7}$ & 0,087 & 0,514 \\
VAR06 & 0,498 & $\mathbf{0 , 5 3 1}$ & 0,224 \\
VAR07 & 0,211 & $\mathbf{0 , 2 3 6}$ & 0,545 \\
VAR08 & 0,529 & $\mathbf{0 , 5 6 2}$ & 0,415 \\
VAR09 & $\mathbf{0 , 5 3 1}$ & 0,299 & 3,737 \\
Total & 4,205 & 3,269 & \\
activo & & & \\
\hline
\end{tabular}

grupos de alumnos) y consigan sanear sus cuentas.

En otras palabras, cada grupo cuenta con un presupuesto inicial de $100 €$. Con ellos, deben abonar cada período $1 €$ por empleado (miembro del grupo). Además, cada fábrica tiene que pagar $10 €$ por cada folio tamaño A4 comprado al gestor de la actividad (el profesor/a responsable de la actividad que también actúa como único cliente del mercado) tal y como se expuso anteriormente al explicar las reglas de la actividad. Por otra parte, los alumnos además de usar los folios que previamente han adquirido deben utilizar las tijeras distribuidas de forma gratuita por el profesor para cortar los folios (la materia prima) según las características de las cartas que se pretenden fabricar.

Las cartas negras tienen un formato alargado siendo posible obtener cuatro cartas por hoja cortándolas en un mismo sentido. Para obtener las cartas rojas se debía cortar la hoja en cuatro partes en dos sentidos distintos. A este respecto, es preciso indicar que el motivo didáctico por el que se pidieron cartas con diferente formato es evitar un corte indiscriminado de determinado tipo de cartas y la formación de un eventual stock de material en proceso por parte de los alumnos (WIP - work in process).

En la etapa de contorno, utilizan los moldes de las cartas distribuidos por el profesor para confeccionar el contorno de las mismas.

El contorno se realiza utilizando bolígrafos rojos o negros según la carta que le corresponda. Por otra parte, en la etapa de pintura los alumnos deben rellenar el contorno realizado en la etapa anterior para lo cual utilizarán los rotuladores (negro o rojo) repartidos por el gestor de la práctica (el profesor), según la carta que deseen fabricar.

A este respecto, es preciso recordar que la demanda del mercado varía cada 120 segundos y que una vez realizado un nuevo pedido (por 


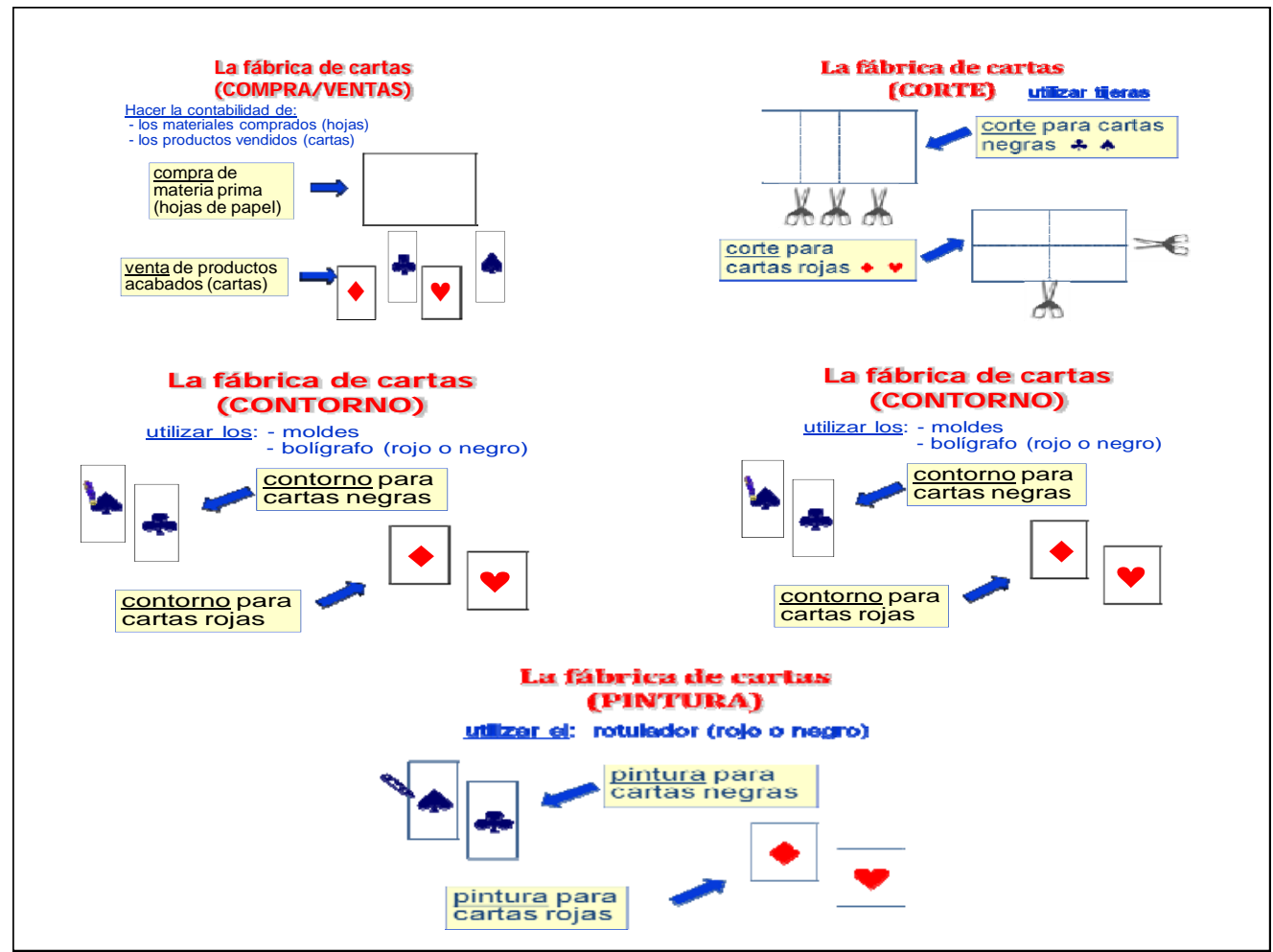

Figura 1: Funcionamiento de la producción de naipes.

ejemplo, en el período 3 se demandan cuatro ases de corazones) el mercado no comprará las cartas que se demandaron durante período anterior (por ejemplo, en el período 2 se demandaban dos ases

de rombos), incluso en el caso en que la demanda no hubiese sido satisfecha durante dicho período por falta de oferta. Esa penalización propició el debate en clase sobre el efecto "Forrester" (efecto látigo) causado por un pedido que continuará en la cadena de abastecimiento pero que ya no será vendido. La Figura 1 resume el proceso de producción llevado a cabo.

Finalmente, tal y como se ha expuesto al explicar las reglas de "La Fábrica de Cartas", durante la etapa de negociación y venta las cartas fabricadas son compradas según su calidad. De este modo, los alumnos saben que el mercado

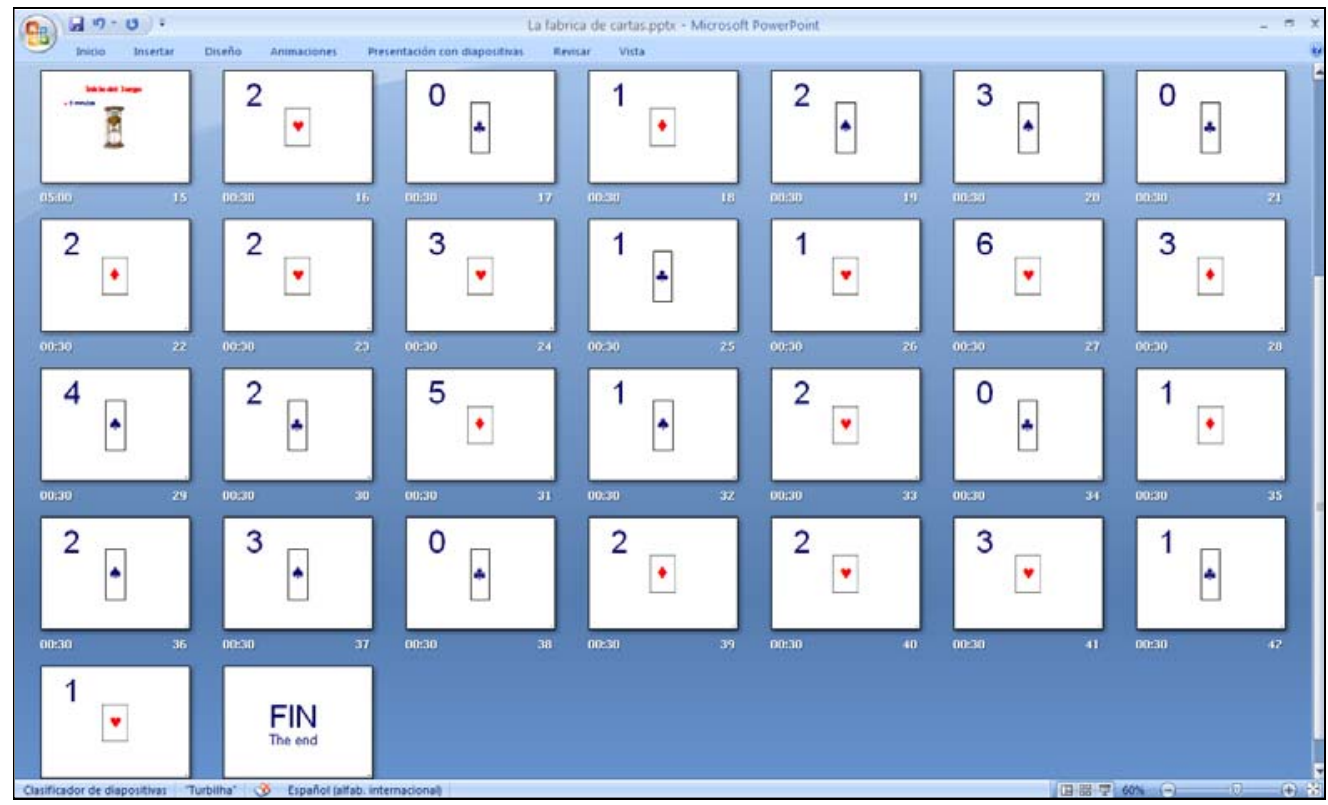

Figura 2.- Simulación de la demanda de naipes. 
está dispuesto a pagar un máximo de $30 €$ por las cartas buenas y un máximo de $15 €$ por las cartas de calidad regular. Las tentativas de "engaño" al mercado, es decir, vender un producto a un precio superior a la calidad que le corresponde, tienen un coste de $10 €$ para cada equipo fabricante. Estos valores - positivos o negativos ayudan a que los estudiantes se percaten de la importancia de la calidad de un producto (bienes y/o servicios) y del modo en que el mercado penaliza a las empresas que entregan productos con calidad inferior a la publicitada. En este sentido, cada grupo fija el precio de la carta y lo escriben en el reverso de las cartas fabricadas. Quien decide la compra es el profesor, ya que además de actuar como gestor de la práctica, actúa como único cliente. De este modo, en función de la demanda de cada período (véase Figura 2) el mercado comprará las cartas más baratas según su categoría de calidad (buena o regular). Gana el equipo que al final de la actividad registre el mejor resultado financiero.

\section{3.- RESULTADOS.}

A través de la práctica descrita en este trabajo, los alumnos tuvieron la oportunidad de integrar de forma activa los conocimientos teóricos adquiridos en diferentes asignaturas de la Licenciatura en Administración y Dirección de Empresas. En concreto, la actividad sirvió para poner en práctica algunos conceptos básicos vinculados al área de producción, gestión empresarial y marketing. En relación a las dos primeras áreas, esta actividad facilitó el debate y análisis en el aula de diversos temas, tales como i) la teoría de las restricciones (identificación y equilibrio de los cuellos de botella); ii) los tipos de layout, con sus ventajas e inconvenientes; iii) el efecto látigo; iv) la previsión de la demanda; v) la importancia de la visión integrada de los subsistemas de la empresa; vi) la importancia de la comunicación eficiente entre los subsistemas y el entorno externo; vii) los procesos de transformación (input, transformación, output); viii) los objetivos de los subsistemas de operaciones (costes, tiempo de entrega, flexibilidad, calidad, y servicio al cliente); ix) la productividad y los conceptos de eficiencia, eficacia $y$ efectividad $y$ finalmente, $x$ ) la definición estratégica de diferenciación o primacía de costes.

Por otra parte, con respecto a los conceptos vinculados al área de marketing aplicados en esta actividad, los alumnos tuvieron la oportunidad de observar de forma práctica cómo incide la demanda del mercado en la fijación de precios para atraer a los clientes y ganar cuota de mercado.
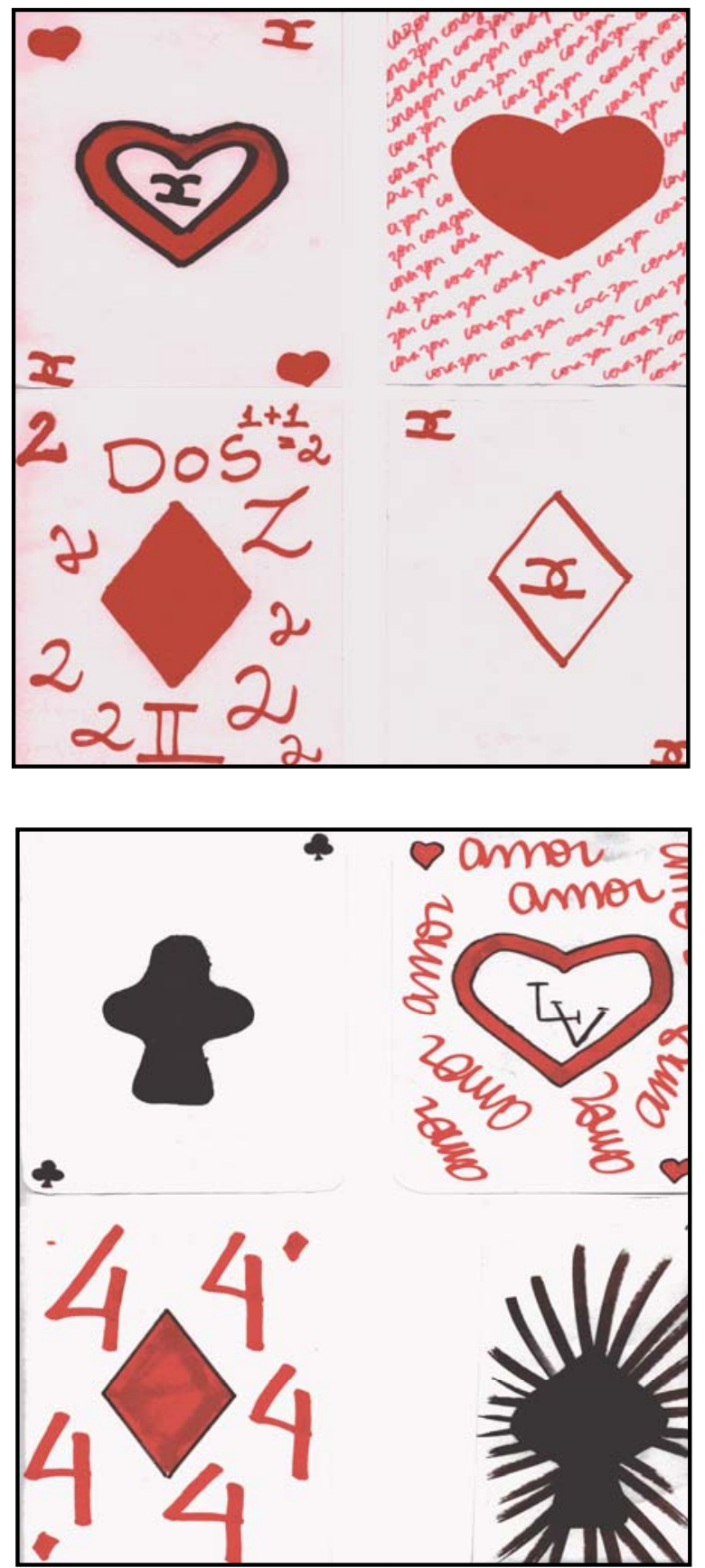

Figura 3.- Ejemplos de diferenciación realizados por el alumnado.

Asimismo, los alumnos entendieron la importancia de ajustar la calidad real del producto con la calidad esperada por los consumidores y con ello, atraer y fidelizar al mercado. De este modo, ya que cada grupo tuvo libertad para producir naipes con la calidad que deseaba y fijar los precios de venta al público, los 
Tabla 3.- Estadísticos descriptivos - segundo cuestionario.

\begin{tabular}{ccccccccc}
\hline VARIABLES & VAR10 & VAR11 & VAR12 & VAR13 & VAR14 & VAR15 & VAR16 & VAR17 \\
\hline Media & 1,467 & 1,667 & 1,511 & 1,756 & 1,667 & 1,689 & 1,622 & 1,533 \\
Desv. típ. & 0,548 & 0,603 & 0,549 & 0,712 & 0,769 & 0,596 & 0,535 & 0,625 \\
Mínimo & 1,000 & 1,000 & 1,000 & 1,000 & 1,000 & 1,000 & 1,000 & 1,000 \\
Máximo & 3,000 & 3,000 & 3,000 & 3,000 & 4,000 & 3,000 & 3,000 & 3,000 \\
\hline
\end{tabular}

alumnos pusieron en práctica políticas de promociones o descuentos temporales. Esto último propició que se desarrollase un debate en clase después de la realización de la actividad

para analizar las condiciones en las que resulta más ventajoso para la empresa (en términos financieros y de reputación de marca) llevar a cabo un descuento en el precio unitario de los productos, o por el contrario, lanzar una promoción que incentive la compra por volumen (promociones de $2 \times 1$, segunda unidad a mitad de precio, etc.)

para incrementar su cuota de mercado y reducir la de sus competidores. Además, los alumnos tuvieron la oportunidad de analizar la idoneidad de la puesta en práctica de estrategias de liderazgo en precios, estrategias de seguimiento y estrategias no sostenibles (overpriced strategies). Por último, tal y como se recoge en la Figura 3, "La Fábrica de Cartas" permitió analizar las circunstancias en las que resulta más eficiente para la empresa adaptar su producto a las necesidades específicas del cliente (customización) a través de la aplicación de diseños novedosos, ensalzar la marca, etc. frente a la estrategia contraria (estandarización).

Una vez llevada a cabo la actividad "La Fábrica de Cartas" y después de haber debatido en clase los conceptos teóricos puestos en práctica, se repartió un cuestionario entre todos los alumnos que participaron en la actividad, donde el anonimato quedaba garantizado. En él se les pedía que evaluase del 1 al 5 - donde 1 indicaba totalmente en desacuerdo y 5 muy de acuerdo - los siguientes aspectos sobre la práctica llevada a cabo ${ }^{4}$ :

1. El tema es aburrido

2. El tema es difícil

3. El profesor es aburrido

4. El material utilizado en clase

\footnotetext{
${ }^{4}$ El lector interesado en el cuestionario completo puede encontrar una copia en el Anexo de este trabajo.
}

\section{Estoy muy cansado}

6. El tema visto en clase no tiene utilidad práctica

7. No entendí los conceptos vistos en clase

8. Me resultó difícil concentrarme en clase

Tal y como se puede observar a partir de los resultados obtenidos, la puesta en práctica de "La Fabrica de Cartas" coadyuvó a la minimización de los inhibidores del aprendizaje identificados en la primera parte de este trabajo. Así pues, en la mayoría de los casos, los alumnos encuestados consideraron que el tema no era aburrido $(71 \%)$, ni difícil (67\%), el profesor no era aburrido (70\%), el material empleado en la actividad no era aburrido (65\%), no estaban muy cansados (67\%), percibieron la utilidad práctica del tema discutido en clase (66\%), entendieron los conceptos vistos en clase (68\%) y finalmente, no les resultó difícil concentrarse en clase $(69 \%)$. La Tabla 3 recoge los estadísticos descriptivos de las variables.

En este sentido, es preciso indicar que los autores hubieran deseado poder llevar a cabo esta actividad con todos los alumnos de $4^{\circ}$ curso de ADE y doble licenciatura con ADE de la Universidad en que trabajan. Sin embargo, debido a la realización de prácticas laborales en horario lectivo, la práctica tuvo que llevarse a cabo con un total de 471 alumnos repartidos en grupos de no más de 40 alumnos por aula. Ello propició la existencia de un mercado con 6-8 empresas fabricantes de cartas en todos los casos. Todos los alumnos que participaron en la actividad contestaron al segundo cuestionario, lo que propició la recogida de 471 cuestionarios válidos aunque hubo dos casos en el que los cuestionarios entregados estaban en blanco. Tal y como se hizo con en el primer cuestionario (destinado a identificar los factores que inhiben el aprendizaje), se calcularon el análisis de correspondencias. En este sentido, los resultados recogidos en la Tabla 4 revelan la existencia de dos dimensiones. La primera de ellas está formada por las variables $10,11,12,13,15$ y 16 
mientras que las variables restantes (14 y 17) componen la segunda dimensión. El Alfa de Cronbach asociado a cada una de las dimensiones es 0,817 y 0,712 respectivamente, lo que permiet descartar la existencia de problemas de multidimensionalidad en cada una de las dos dimensiones identificadas. Ello implica que el cuestionario estuvo libre de error aleatorio y por lo tanto, proporcionaría resultados prácticamente idénticos en todos los casos en que se repitiera la investigación (Cronbach, 1951).

Por otra parte, junto a los ocho ítems antes expuestos, el cuestionario incluía varías preguntas abiertas a través de las cuales se les solicitaba a los alumnos que expresaran su opinión general acerca de la actividad llevada a cabo ("La Fábrica de Cartas"). En este sentido, el 92 por ciento consideró que se trataba de una experiencia enriquecedora y que les había servido para integrar los conocimientos adquiridos durante el curso.
Asimismo, se les preguntó qué era lo que más les había gustado de la actividad y la mayoría destacó el trabajo en equipo, el cambio que supuso en la manera de aprender los conceptos, la interactividad con el profesor y los compañeros, así como la posibilidad de desenvolverse en situaciones reales de mercados competitivos. Sin embargo, el 8\% de los alumnos manifestaron, por ejemplo, que "me compliqué demasiado en resolverla, fue divertida e interesante, me gustó la idea de simular un mercado real pero las otras empresas bajaron mucho los precios y no tuve oportunidad de vender mis cartas". Junto a ello, algunos estudiantes expresaron que la práctica les había ayudado mucho para entender los conceptos teóricos vistos en clase pero "se habían manchado las manos con los rotuladores al fabricar las cartas".

Tabla 4.- Resultados del análisis de correspondencias asociado al segundo cuestionario.

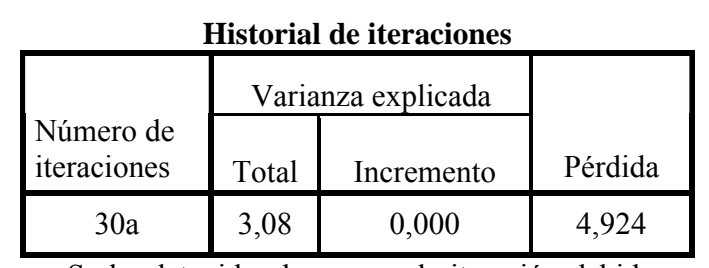

a. Se ha detenido el proceso de iteración debido a que se ha alcanzado el valor de la prueba para la convergencia.

\begin{tabular}{|c|c|c|c|}
\hline \multicolumn{2}{|c|}{ Medidas de discriminación } \\
\cline { 2 - 3 } & \multicolumn{2}{|c|}{ Dimensión } & \multirow{2}{*}{ Media } \\
\cline { 2 - 3 } & 1 & 2 & 0,446 \\
VAR10 & $\mathbf{0 , 5 5 3}$ & 0,338 & 0,367 \\
VAR11 & $\mathbf{0 , 3 7 7}$ & 0,357 & 0,564 \\
VAR12 & $\mathbf{0 , 7 2 3}$ & 0,404 & 0,535 \\
VAR13 & $\mathbf{0 , 5 9 8}$ & 0,472 & 0,421 \\
VAR14 & 0,330 & $\mathbf{0 , 5 1 2}$ & 0,173 \\
VAR15 & $\mathbf{0 , 2 4 8}$ & 0,099 & 0,289 \\
VAR16 & $\mathbf{0 , 3 9 6}$ & 0,182 & 0,282 \\
VAR17 & 0,272 & $\mathbf{0 , 2 9 2}$ & 3,076 \\
Total activo & 3,496 & 2,655 & 0,446 \\
\hline Total activo & $\mathbf{, 5 5 3}$ & 0,338 & \\
\hline
\end{tabular}

\begin{tabular}{|c|c|c|c|}
\hline \multirow{2}{*}{ Dimensión } & \multirow{2}{*}{ Resumen del modelo } \\
\cline { 3 - 4 } & Alfa de & \multicolumn{2}{|c|}{ Varianza explicada } \\
\cline { 3 - 4 } & Cronbach & $\begin{array}{c}\text { Total } \\
\text { (Autovalores) }\end{array}$ & Inercia \\
\hline 1 & $\mathbf{0 , 8 1 6}$ & 3,496 &, 437 \\
2 & $\mathbf{0 , 7 1 2}$ & 2,655 &, 332 \\
Total & & 6,151 &, 769 \\
Media & $0,771^{\text {a }}$ & 3,076 &, 384 \\
\hline
\end{tabular}

a. El Alfa de Cronbach Promedio está basado en los autovalores promedio. 


\section{4.- CONCLUSIONES.}

No son muchos los trabajos realizados sobre innovación en los procesos de aprendizaje (Baena et al., 2008). Además, los resultados obtenidos en los estudios que han abordado este tema no pueden generalizarse a todo el sistema educativo ya que en la mayoría de los casos dichos trabajos se han llevado a cabo con estudiantes de universidades norteamericanas y británicas pertenecientes a titulaciones técnicas o de ciencias de la salud (véase, por ejemplo, Hunt, Eagle y Tchen, 2004; Meterissian, Liberman y McLeod, 2007).

Este artículo pretende contribuir a la literatura mediante la susbsanación de estas limitaciones. Para ello, se presenta una actividad llevada a cabo en la Facultad de Ciencias Sociales de una Universidad española mediante la cual se integraron varios conceptos de las áreas de producción, gestión de empresas y marketing que adquieren los estudiantes de la Licenciatura en Administración y Dirección de Empresas. Esto facilitó que los alumnos adquiriesen una visión integradora de numerosos contenidos analizados a lo largo de la carrera y se lograran resultados congruentes a los objetivos estratégicos establecidos en Bolonia para el EEES. Por todo ello, nos atrevemos a afirmar que "La Fábrica de Cartas" contribuyó a que los alumnos adoptasen un aprendizaje activo y su motivacion en el aula aumentase. Además, durante el desarrollo de la actividad los alumnos mostraron una actitud de competitividad sana y colaborativa con sus compañeros, así como responsable con respecto al resto de los miembros de su grupo de trabajo.

Los autores no tienen conocimiento de ningún trabajo publicado en la literatura que identifique algunos de los factores que inhiben el aprendizaje y a su vez, propongan una actividad docente que ayude a minimizar dichos efectos. Precisamente, creemos que una de las principales contribuciones a la literatura dimana de este hecho. Sin embargo, este estudio pretende, además, proporcionar diferentes implicaciones prácticas para las personas involucradas en el diseño de los planes de estudio de Grado de nuestras universidades. De este modo, confiamos en que los resultados obtenidos en este trabajo sirvan tanto a los profesores como coordinadores/directores de titulación para determinar futuras acciones y desarrollar planes estratégicos que minimicen los inhibidores del aprendizaje.

Una de las principales limitaciones de este trabajo es que la muestra sólo incluye alumnos de $4^{\circ}$ curso de ADE y doble licenciatura con ADE (ADE + Investigación y Técnicas de Mercado, ADE + Publicidad, ADE + Ingeniería Industrial, $\mathrm{ADE}+$ Ingeniería Informática, así como $\mathrm{ADE}+$ Ingeniería de Telecomunicaciones) impartidas en la universidad donde trabajan los autores. En este sentido, y como futura línea de investigación, sería interesante que se replicase este trabajo en otras universidades y con alumnos de otras titulaciones para comparar los resultados obtenidos. Ello nos permitiría contrastar si las conclusiones alcanzadas en este estudio pueden ser generalizados a cualquier tipo de alumnado sin importar su área de conocimiento y la universidad donde estudian.

Finalmente, los cambios a los que nos enfrentamos en el EEES hacen necesario que las universidades profundicen en la necesidad de conocer a su alumnado y adapten no sólo sus programas formativos sino también, las metodologías docentes empleadas en los nuevos títulos de grado. Por ello, esperamos que futuros trabajos puedan utilizar este estudio como punto de partida para identificar nuevas variables capaces de inhibir el aprendizaje de nuestros alumnos, así como el tipo de actividades que les pueden ayudar - a la vez que estimular -, en el desarrollo de algunas competencias y habilidades necesarias en el mundo laboral.

\section{5.- REFERENCIAS BIBLIOGRÁFICAS.}

Adrian, G. M. \& Palmer, G. D. (1999). Toward a Model for Understanding and Improving Educational Quality in the Principles of Marketing Course. Journal of Marketing Education, 21(1), 25-33

Baena, V., Figueroa, C., Gabaldón, P., García-Viana, R., Gava, L., Hernán, R., López, C. \& Priede, T. (2008). El espacio europeo de educación superior: Una oportunidad para la innovación educativa en el área empresarial. Madrid: Universidad Europea de Madrid.

Barber, M. (2008). A formula for great teaching. Times Educational Supplement, 48, 19-29.

Barreiros, B., Díez, E., Díez, J.T., Rondán, F.J. \& Sánchez, M. J. (2004). La elección del canal de distribución comercial. Madrid: McGraw-Hill.

Bullock, K. \& Muschamp, Y. (2006). Learning about learning in the primary school. Cambridge Journal of Education, 36(1), 49-62. 
Canzer, B. (1997). Marketing education on the internet: a world wide web based introductory marketing course design for the virtual-u project in distance education at Simon Fraser University. Journal of Marketing Education, 23(1), 56-65.

Christensen, C. M., Horn, M.B. \& Johnson, C.W. (2008). How 'disruptive innovation' will change the way we learn. Education Week, 27(39), 25-36

Cronbach, L. J. (1951). Coefficient Alpha y the internal structure of tests. Psychometrika, 16(Sep), 297-334

Cuestas, P., Fenollar P. \& Román, S. (2008). ¿Aprenden nuestros alumnos? Influencia de las variables y la infraestructura docente en el aprendizaje, Actas del XX. Actas del XX Encuentro de Profesores Universitario de Marketing, Gran Canarias (España).

De Juan, M. D., González, E., Parra, J. F., Kanther, A. \& Sarabia, F. J. (2008) Antecedentes del aprendizaje autorregulado del estudiantes universitario de marketing. Actas del $X X$ Encuentro de Profesores Universitario de Marketing, Gran Canarias (España).

Delors, J. (1998). La educación encierra un tesoro Colombia. UNESCO-Santillana.

Elliott, K. M. \& Shin, D. (2002). Student satisfaction: an alternative approach to assessing this important concept. Journal of Higher Education Policy \& Management, 24(2), 197-209

Eyler, G.N. \& Cañizo, A.I. (2006). Actividad integradora de conocimientos, capacidades y habilidades en química orgánica, Actas de las VII Jornadas de Enseñanza Universitaria de la Química, Comodoro Ribadavia, Argentina.

García-Aracil, A. (2009). European graduates' level of satisfaction with higher education. Higher Education, 57(1), 1-21

Gibbs, P. (2007). Editorial, Journal of Business Research, 60(9), 925-926.
Hair, J., Anderson, R. E., Tatham, R. \& Black, W. C. (1999). Análisis multivariante ( $5^{\mathrm{a}}$ edición). Madrid: Prentice-Hall International, Inc.

Hunt, L., Eagle, L., \& Tchen, P (2004). Balancing marketing education $\mathrm{y}$ information technology: Matching needs or needing a better match? Journal of Marketing Education, 26(1), 75-88.

López, M. (2008). La integración de las Habilidades Sociales en la escuela como estrategia para la salud emocional, Revista Electrónica de Intervención Psicosocial y Psicología Comunitaria, 3(1), 16-19

Meterissian, S., Liberman, M. \& Mcleod, P. (2007). Games as teaching tools in a surgical residency. Medical Teacher, 29(9/10), 258-270

Norman, M. \& Hyland, T. (2003). The Role of Confidence in Lifelong Learning. Educational Studies, 29(2/3), 261-273.

Pagani, R. \& González, J. (2002). El crédito europeo y el sistema educativo español. Informe técnico ECTS. Counsellors y Diploma Suplement Promoters.

Peterson, A. R. (1994). A meta-analysis of Cronbach's coefficient alpha. Journal of Consumer Research, 21(2), 381-391

Pusztai, G. \& Szabó, P. C. (2008). The Bologna process as a Trojan horse. European Education, 40(2), 85-103.

Shu-Hui, H.C. \& Smith, R.A. (2008). Effectiveness of personal interaction in a learner-centered paradigm distance education class based on student satisfaction. Journal of Research on Technology in Education, 40(4), 407-426.

Whitehead, D.P. (2008). Thoughts on Education and Innovation. Childhood Education, 85(2), 106-118

Wright, L.K., Bitner, M.J. \& Zeithaml, V.A. (1994). Paradigm shifts in business education: using active learning to deliver services marketing content. Journal of Marketing Education, 16(Fall), 5-19.

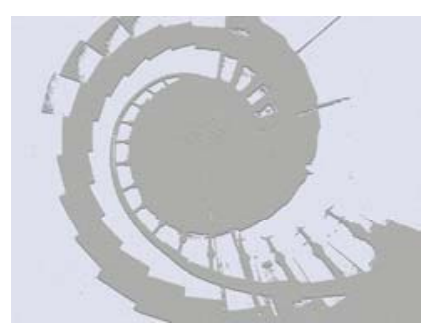

